\title{
Analysis of anisotropy of ultrahigh energy cosmic ray arrival direction and energy spectrum detected by Telescope array experiment
}

\section{Wooram Cho'}

Hanyang University

441 Science bldg. Hanyang University, Wangsipri-ro 222, Sungdong-gu, Seoul, South Korea

E-mail: wrcho@hanyang.ac.kr

\section{Eunho Lee}

Hanyang University

441 Science bldg. Hanyang University, Wangsipri-ro 222, Sungdong-gu, Seoul, South Korea

\section{Hang Bae Kim}

Hanyang University

441 Science bldg. Hanyang University, Wangsipri-ro 222, Sungdong-gu, Seoul, South Korea

abstract

Ultra high energy cosmic rays over $10^{\wedge} 19 \mathrm{eV}$ are thought to come from very powerful objects such as Active Galatic Nucleus(AGN). However, the number of AGNs and ultra high energy cosmic rays is not large enough to experimentally declare the correlation between them. We use Watson and Mortlock's bayesian statistical method to compare the arrival direction data of large ground arrays such as the Telescope Array experiment with AGNs in the Veron-Cety and Veron (VCV) catalog. We also test the linearity using toy monte carlo simulation. In this presentation, we present the probability that the TA's cosmic rays come from AGNs.

36th International Cosmic Ray Conference -ICRC2019-

July 24th - August 1st, 2019

Madison, WI, U.S.A.

\section{${ }^{1}$ Speaker}




\section{Introduction}

Telescope Array project is the experiment to observe the Ultra-high energy cosmic rays (UHECRs) located in Utah, USA operating since 2008. The experiment is consisted of two detector, Surface detector(SD) and Fluorescence cdetector(FD) which observe the extensive air showers(EAS) of UHECRs.

TA reported a possible point source, Hotspot. If there are point sources like hotspot, but with low energy, the energy spectrum around the source direction can be different from other direction. We study energy spectrum of UHECRs with energy over 10EeV on TA's field of view(FOV), using 9 years data of TASD.

\section{Method}

Analysis procedures are like the followings.

Scan the TA exposure with spherical cap.

To avoid a bias that depends on declination,

make spherical cap have a constant exposure ratio, $\alpha=N_{\text {on }} / N_{\text {off }}$

Construct energy histogram with events inside a cap.

$\rightarrow$ observed energy bin frequencies $\left(n_{i}\right)$

Make another energy histogram with events outside the cap that normalized with expected number of events inside the $\operatorname{cap}\left(N_{b g}=\alpha\left(N_{\text {events }}-N_{\text {on }}\right)\right)$

$\rightarrow$ expected energy bin frequencies $\left(\mu_{i}\right)$

If the expected number of events in an energy $\operatorname{bin}\left(\mu_{i}\right)$ is smaller than 1 , the bin are combined with adjacent bins to reduce a bias.

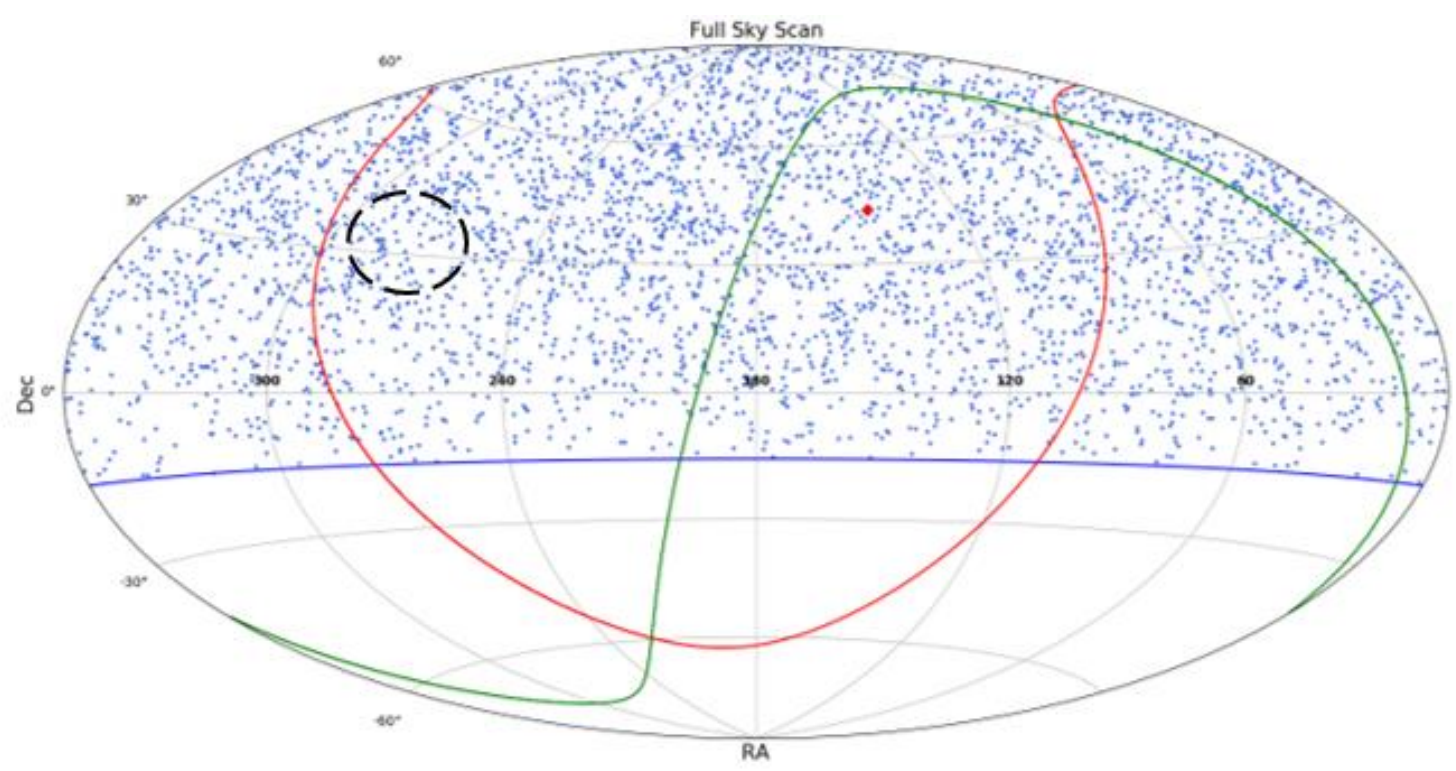

- Calculate a significance for a cap by using the binned Poisson likelihood ratio test. 
Likelihood ratio test is a test which consider a ratio of likelihood of null hypothesis to that of alternative one as statistic.

$$
\left(\Lambda=L\left(\theta_{0} \mid x\right) / L\left(\theta_{\text {alt }} \mid x\right)\right.
$$

If a ratio is too small, the null hypothesis is rejected.

If the sample size approaches to $\infty$, the test statistic $(-2 \log (\Lambda))$ is approximately chisquared distributed $\left(\chi^{2}\right)$ : Wilks' theorem

Final test statistic is

$$
-2 \log (\Lambda) \cong \chi^{2}
$$

$$
\chi^{2} \cong 2 \sum_{i}\left(\mu_{i}-n_{i}+n_{i} \ln \left(n_{i} / \mu_{i}\right)\right)
$$

The degrees of freedom are

$$
\text { dof }=\text { number of bins }+2
$$

The estimated background and the rebinning give additional degrees of freedom

Likelihood ratio test furnishes quantitative statistic how different two histograms are.

However, the statistic does not tell us any physical difference of two histograms. To see this, we decide to fit the most significant histograms with broken power law function.

\section{Results}

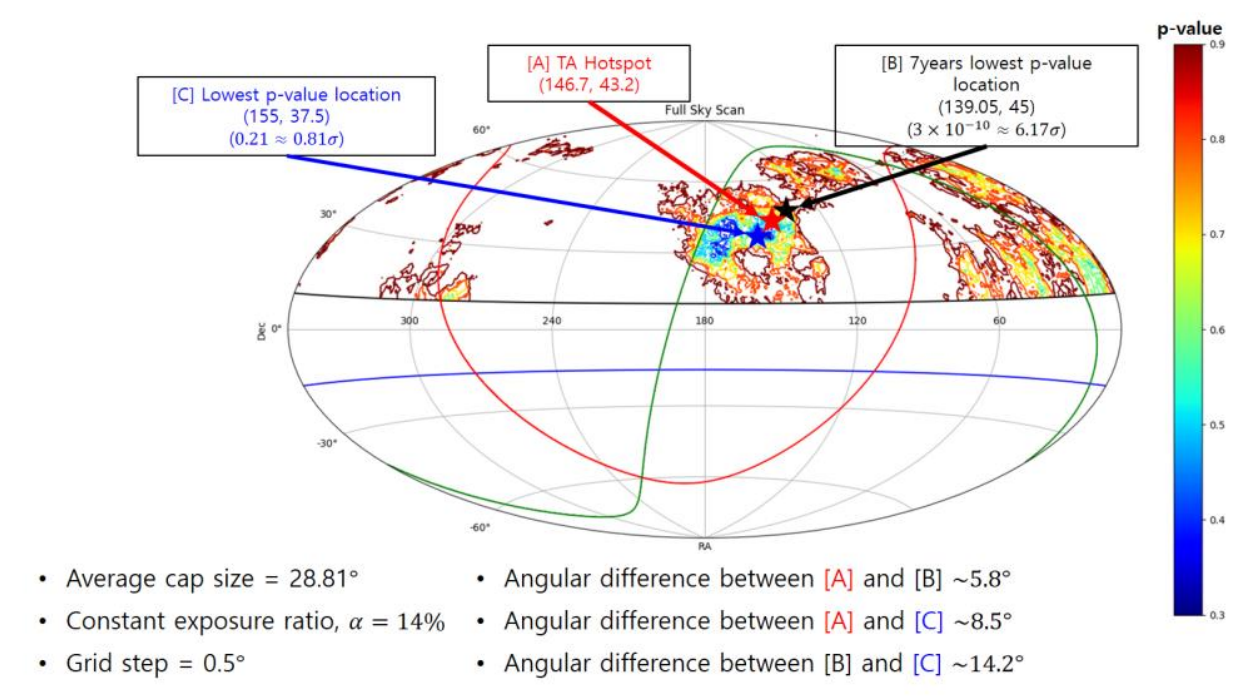



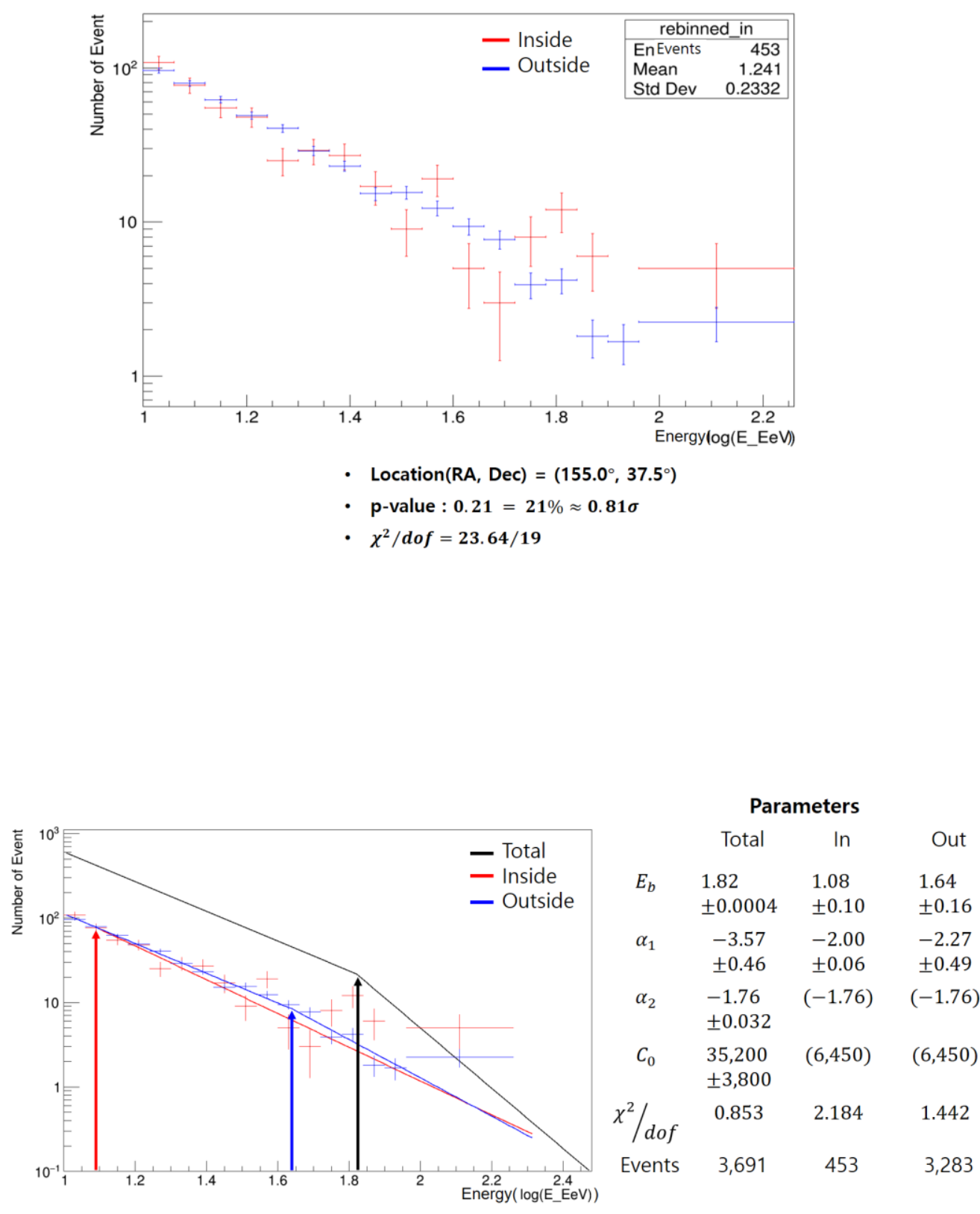

$$
N(E) / \log _{10}\left(\frac{E}{1 E e V}\right)=C_{0}\left(\theta\left(E_{b}-E\right)\left(\frac{E}{1 E e V}\right)^{-\alpha_{2}}+\theta\left(E-E_{b}\right)\left(\frac{E_{b}}{1 E e V}\right)^{-\alpha_{2}+\alpha_{1}}\left(\frac{E}{1 E e V}\right)^{-\alpha_{1}}\right)
$$

\section{Discussion}

Significance is decreased in comparison with that using 7years data. There is one different factor, which is the energy threshold. Adjusting the threshold means that changing the energy scale of anisotropy. Thus, we cannot see the energy spectrum anisotropy in $10 \mathrm{EeV}$ energy scale by using Poisson likelihood ratio test. The rebinning process makes histogram fluctuate so histograms look like coming from similar distribution. Therefore, an additional process such as fitting the histograms should be performed to claim both the statistical difference of histograms 
and the physical difference of those. We used Poisson likelihood ratio test to find an energy spectrum anisotropy with energy scale $10 \mathrm{EeV}$ using 9years data of TASD. The most significant histograms are located at $\left(155.0^{\circ}, 37.5^{\circ}\right)$ with p-value, 0.21 which is decreased compared with former result. Thus, we conclude that we cannot see the energy spectrum anisotropy in $10 \mathrm{EeV}$ scale. Moreover, we tried to fit the most significant histograms to see how physically different two histograms are. The fitted energy histograms are not good because of low number of events. However, fitting the histograms may be needed to claim that the histograms are different physically.

\section{References}

[1] Abbasi, R. U., et al. Evidence of Intermediate-scale Energy Spectrum Anisotropy of Cosmic Rays E $\geq 10^{19.2} \mathrm{eV}$ with the Telescope Array Surface Detector. The Astrophysical Journal 862.2 (2018): 91.

[2] Abbasi, R.U., et al. Indications of intermediate-scale anisotropy of cosmic rays with energy greater than 57EeV in the northern sky measured with the surface detector of the telescope array experiment. The Astrophysical Journal Letters 790.2 (2014): L21.

[3] Abbasi, R. U., et al. Search for Anisotropy in the Ultra High Energy Cosmic Ray Spectrum using the Telescope Array Surface Detector. arXiv preprint arXiv:1707.04967 (2017).

[4] Baker, Steve, and Robert D. Cousins. Clarification of the use of chi-square and likelihood functions in fits to histograms. Nuclear Instruments and Methods in Physics Research 221.2 (1984): 437-442. 